\title{
The role of detecting BRAF T199A mutation in fine-needle aspiration biopsy in pre-operative diagnosis of nodular goiter.
}

Anhelli Syrenicz, Monika Koziołek ${ }^{1}$, Bartosz Kiedrowicz' ${ }^{1}$ Miłosz Parczewski ${ }^{2}$, Maria Stepaniuk $^{3,6}$,
Elżbieta Andrysiak-Mamos ${ }^{1}$, Joanna Obłoza ${ }^{4}$, Anna Sieradzka ${ }^{1}$, Agnieszka Bińczak-Kuleta ${ }^{5}$

POMERANIAN MEDICAL UNIVERSITY, SZCZECIN, POLAND

(1) Department of Endocrinology, Metabolic and Internal Diseases,

(2) Department of Infectious Diseases, Hepatology and Immune Deficiency,

(3) Department of Pathology,

(4) Department of Endocrinology, M.Curie Regional Hospital, Szczecin, Poland

(5) Department of Laboratory Diagnostics and Molecular Medicine,

(6) Department of Surgery, West Pomeranian Oncology Center, Szczecin, Poland

\section{INTRODUCTION \&} OBJECTIVES

Molecular testing plays increasingly a significant role in the pre-operative diagnosis of nodular goiter, as standard methods may not give conclusive decision in choosing optimal treatment approach. BRAF gene mutations are often discovered in the cytological specimens among the patients with papillary thyroid cancer.

The aim of the study was to assess the incidence of BRAF T1799A mutation in cellular specimens derived from fine needle aspiration biopsy (FNAB) of thyroid nodules.

\section{METHODS}

- 85 women with nodular goiter were enrolled into the study.

- Using hormonal tests, autoantibodies and ultrasound, both hormonal thyroid dysfunction and autoimmune process were excluded.

- All the patients underwent FNAB of revealed nodules.
- We analyzed genomic DNA isolated from the thyroid lesions and peripheral blood.

- Standard methods of real-time amplification detection (real-time PCR) were used to analyze BRAF mutation, with the use of specific starters surrounding the mutated site.

\section{RESULTS}

- We found BRAF T1799A mutation in thyroid specimens of $6(7.05 \%)$ subjects.

- In 5 of them, benign nature of the thyroid nodules was confirmed by FNAB of the lesions.

- This procedure was non-diagnostic in 1 subject and histopathology post-operative assessment confirmed papillary thyroid cancer.

\section{CONCLUSIONS}

The presence of BRAF mutation in FNAB cytological specimens of benign thyroid nodules may be useful to evaluate the risk of malignancy, support the diagnosis and choose treatment options of nodular goiter.

ECE 2015 - DUBLIN 16-20th May 2015

TOPIC: THYROID NON-CANCER
Project supported by the research grant from the Polish Ministry of Science and Higher Education (grant number N N402466739) 